\title{
Overwhelming rapid metabolic and structural response to apatinib in radioiodine refractory differentiated thyroid cancer
}

\author{
Yansong Lin ${ }^{1, *}$, Chen Wang ${ }^{1, *}$, Wen Gao ${ }^{2}$, Ruixue Cui ${ }^{1}$ and Jun Liang ${ }^{3}$ \\ ${ }^{1}$ Department of Nuclear Medicine, Peking Union Medical College Hospital, Beijing 100730, China \\ ${ }^{2}$ Department of Oncology, The Affiliated Hospital of Qingdao University, Qingdao 266555, China \\ ${ }^{3}$ Department of Oncology, Peking University International Hospital, Beijing 102206, China \\ *These authors have contributed equally to this work \\ Correspondence to: Yansong Lin, email: linys@pumch.cn \\ Jun Liang, email: liangjun1959@aliyun.com \\ Keywords: apatinib, radioiodine refractory differentiated thyroid cancer, thyroglobulin, maximum standard uptake value, safety \\ Received: September 26, $2016 \quad$ Accepted: January 08, $2017 \quad$ Published: February 02, 2017 \\ Copyright: Lin et al. This is an open-access article distributed under the terms of the Creative Commons Attribution License 3.0 (CC BY 3.0), \\ which permits unrestricted use, distribution, and reproduction in any medium, provided the original author and source are credited.
}

\section{ABSTRACT}

Currently, patients with radioiodine refractory differentiated thyroid cancer (RAIRDTC) have limited treatment options. In this study, we aimed to assess the short-term efficacy and safety of apatinib in RAIR-DTC. Ten adult patients were prospectively enrolled to receive oral apatinib (750 $\mathrm{mg}$ q.d). The primary endpoints were change in serum thyroglobulin ( $\mathrm{Tg}$ ) concentration, disease control rate (DCR) and objective response rate (ORR) based on RECIST 1.1 criteria. The secondary endpoints included change in glucose metabolism, evaluated by maximum standard uptake value (SUVmax), and safety. As early as 2 weeks after apatinib treatment, the serum $\mathrm{Tg}$ concentration decreased by $21.0 \%$ in 8 patients available for detection without interference, and a further sharp decline by $\mathbf{8 1 . 4 \%}$ compared with the baseline level occurred at 8 weeks post-treatment. The DCR and ORR were $100 \%(10 / 10)$ and $90 \%(9 / 10)$, respectively. The sum of tumor diameter shrank to $22.8 \pm 8.1 \mathrm{~mm}$ from $38.8 \pm 15.7 \mathrm{~mm}(P=0.001)$. Moreover, a significant decrease in SUVmax was observed from $6.53 \pm 5.14$ to $2.56 \pm 1.67$ and $2.45 \pm 1.48$ at 4-week and 8-week time-points after treatment $(P=0.032$ and 0.020$)$, respectively. The common grade 3 adverse events (AEs) included hand-foot-skin reaction (50\%), hypertension $(30 \%)$, and hypocalcemia $(20 \%)$. No severe AE related to apatinib was observed during treatment. Hence, apatinib seems to be a promising therapeutic option for RAIR-DTC patients. Apart from RECIST 1.1 criteria, the biochemical marker ( $\mathrm{Tg}$ ) and glucose metabolism index (SUVmax) could be adopted in assessing the early response to TKI in RAIR-DTC.

\section{INTRODUCTION}

Differentiated thyroid cancer (DTC) arises from aberrant follicular cells. It accounts for nearly $95 \%$ of all thyroid neoplasm, with papillary thyroid cancer (PTC) comprising $90 \%$ of the DTC [1]. With a rapidly rising incidence especially of PTC in recent decades, DTC has become a global concern [2,3]. Usually, most patients with DTC could achieve good prognoses following surgery, radioactive iodine (RAI) therapy, or thyroxin therapy [4]. However, $1-23 \%$ of DTC patients unfortunately develop distant metastases, $30 \%$ of whom will progress to radioiodine refractory DTC (RAIR-DTC) $[5,6]$. With a 10 -year survival rate less than $10 \%$, RAIR-DTC is the major cause of thyroid cancer-related death [6, 7]. Recent discoveries in the molecular mechanisms implicated in RAIR-DTC have provided insight of its pathogenesis and progression $[8,9]$. For example, $\mathrm{BRAF}^{\mathrm{V} 600 \mathrm{E}}$ mutation has been reported to be associated with non-radioiodine-avid status in distant metastatic lesions, and might account for the mechanism of RAIR-PTC [10]. Besides, a range of $39.5 \%$ to $100 \%$ of vascular endothelial growth factor (VEGF) expression also indicated the possible implication of VEGF and its related pathway in the course of DTC gradually becoming refractory to RAI therapy [11-13]. Our earlier study using ${ }^{99 \mathrm{~m}} \mathrm{Tc}-3 \mathrm{PRGD} 2$ targeting integrin $\alpha \mathrm{v} \beta 3$ 
imaging further indicated an in-vivo up-regulation of angiogenesis in lesions of RAIR-DTC [14]. All these findings provided evidences for the potential indication of VEGF receptor (VEGFR) inhibitor in RAIR-DTC [15].

Apatinib is a small-molecule tyrosine kinase inhibitor (TKI) that highly and selectively inhibits VEGFR-2, leading to inhibition of VEGF-mediated endothelial cell migration and proliferation and decrease in tumor microvascular density. Previous studies have proven the potent efficacy and acceptable safety profile of apatinib in gastric cancer $[16,17]$. However, there has been no report regarding its use in thyroid cancer so far. This pilot study firstly focused on the early efficacy evaluation of apatinib in RAIR-DTC. Along with the response evaluation in terms of Response Evaluation Criteria in Solid Tumors (RECIST), thyroglobulin (Tg) monitoring and positron emission tomography / computed tomography (PET/CT) scan were also applied to identify the early biochemical and lesion's glucose metabolic response to apatinib.

\section{RESULTS}

\section{Patient}

A total of 10 patients were included in this study (Figure 1), aged from 32 to 76 years with a mean of 54.9 years. The male-to-female ratio was 1:1. All patients underwent total or near-total thyroidectomy and lymph node (LN) resection and were pathologically confirmed as PTC. Nine of the 10 patients lost the ability of RAI uptake in metastatic lesions at the first RAI treatment after successful remnant ablation. The Tg levels ranged from 0.43 to $7591 \mathrm{ng} / \mathrm{mL}$ at baseline. All participants received apatinib treatment and thus were included in the efficacy and safety analyses. All of the target lesions met the RECIST 1.1 criteria were pulmonary metastatic lesions. The baseline characteristics of the enrolled patients are shown in Table 1.

\section{Efficacy}

\section{Evaluation of early biochemical response}

Eight out of the 10 patients were available for early biochemical response evaluation, while the remaining 2 patients were excluded from analysis due to interference with high-level thyroglobulin antibody (TgAb) (>4000 $\mathrm{IU} / \mathrm{mL}$ ) in $\mathrm{Tg}$ detection or low levels of $\mathrm{Tg}$ at baseline and during treatment $(<1 \mathrm{ng} / \mathrm{mL})$. The Tg concentration increased by $41.0 \%$ within the 12 months before apatinib therapy, but decreased by $40.2 \%$ after apatinib administration (the individual responses are shown in Figure 2A). In fact, as early as 2 weeks after therapy, a

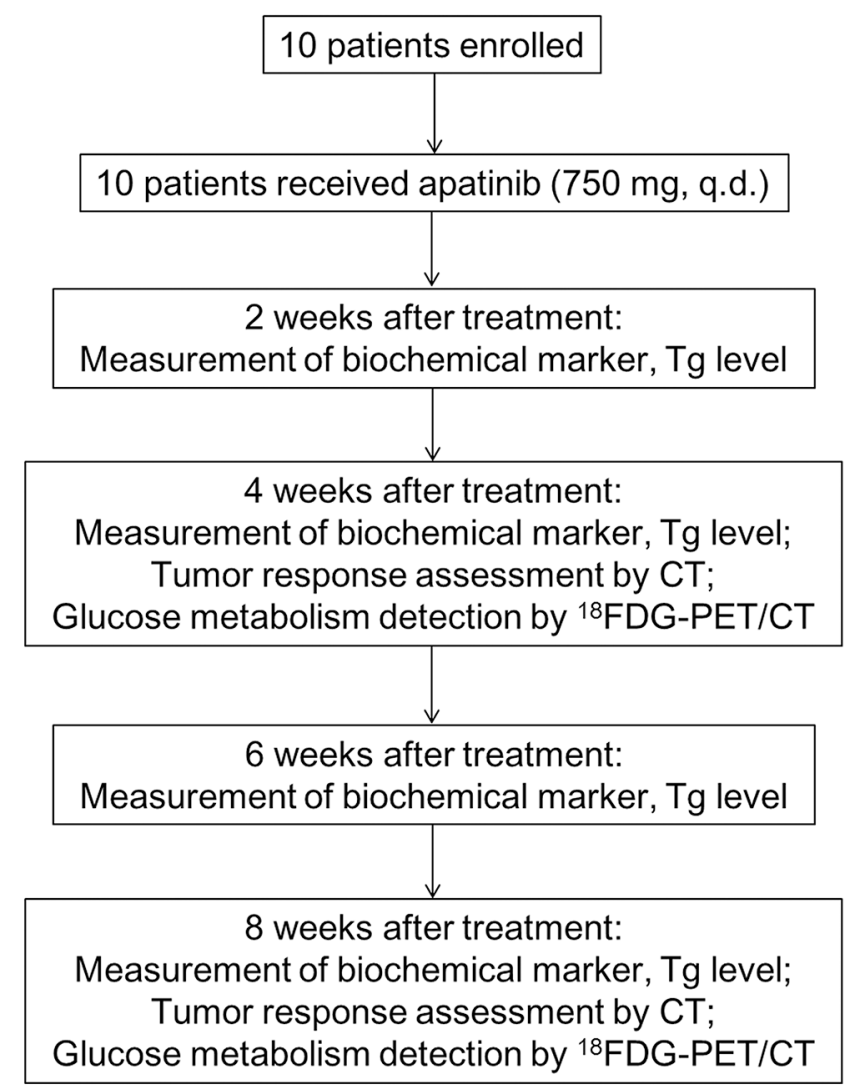

Figure 1: Study diagram. 
Table 1: The baseline characteristics of enrolled patients and tumor response 8 weeks after apatinib treatment

\begin{tabular}{|c|c|c|c|c|c|c|c|c|c|}
\hline No. & Gender & Age & Pathology & TNM stage & Metastasis site & VEGF & $\begin{array}{c}\text { Accumulated } \\
\text { RAI dose (mCi) }\end{array}$ & $\begin{array}{l}\text { RAI* } \\
\text { uptake }\end{array}$ & Response \\
\hline 1 & M & 44 & PTC & pT3N1bM1 & $\begin{array}{c}\text { Pulmonary \& } \\
\text { head }\end{array}$ & - & 300 & No & PR \\
\hline 2 & M & 47 & PTC & pTxN1aM1 & $\begin{array}{c}\text { Pulmonary \& } \\
\text { bone }\end{array}$ & - & 305 & No & PR \\
\hline 3 & M & 76 & PTC & pT4aN1bM1 & Pulmonary & + & 300 & No & PR \\
\hline 4 & $\mathrm{~F}$ & 32 & PTC & pT4aN0M1 & Pulmonary & - & 200 & No & SD \\
\hline 5 & M & 51 & Poorly-PTC & pT4aNxM1 & Pulmonary & + & 350 & No & PR \\
\hline 6 & $\mathrm{~F}$ & 75 & PTC & pT4aN1bM1 & $\begin{array}{c}\text { Pulmonary \& } \\
\text { bone }\end{array}$ & - & 230 & No & PR \\
\hline 7 & M & 65 & PTC & pT4aN1bM1 & Pulmonary & N/A & 350 & No & PR \\
\hline 8 & $\mathrm{~F}$ & 46 & PTC & pT3N1bM1 & $\begin{array}{c}\text { Pulmonary \& } \\
\text { bone }\end{array}$ & - & 630 & No & PR \\
\hline 9 & $\mathrm{~F}$ & 63 & PTC & pT4aN1bM1 & Pulmonary & + & 325 & Yes & PR \\
\hline 10 & $\mathrm{~F}$ & 49 & PTC & pT4aN1bM1 & Pulmonary & - & 440 & No & PR \\
\hline
\end{tabular}

VEGF: vascular endothelial growth factor; RAI: radioactive iodine; PTC: papillary thyroid cancer; PR: partial response; SD: stable disease;

N/A: not available.

*RAI uptake in metastasis lesions at the first RAI therapy.

decline by $21.0 \%$ in $\mathrm{Tg}$ could be noticed, indicating a rapid biochemical response to apatinib. Furthermore, the $\mathrm{Tg}$ concentration was continually decreased by $81.4 \%$ from the baseline level (from $1583.5 \mathrm{ng} / \mathrm{mL}$ at baseline to 294.6 $\mathrm{ng} / \mathrm{mL}$ at 8 -week time-point). Figure $3 \mathrm{~A}$ shows a sharp decline in Tg level in a 76-year-old male patient. Unlike other cases, one 46-year-old female patient did present an increase in Tg level from $7591.0 \mathrm{ng} / \mathrm{mL}$ to $11191.0 \mathrm{ng} / \mathrm{mL}$ 2 weeks after therapy; however, the $\mathrm{Tg}$ level decreased to $2947.0 \mathrm{ng} / \mathrm{mL} 4$ weeks after treatment.

\section{Evaluation of target lesions response according to RECIST 1.1}

Eighteen target lesions of the 10 patients were identified and evaluated. The growth rate was 1.38 $\pm 0.98 \mathrm{~mm}$ per month within the 12 months before apatinib treatment, and the total tumor diameter (TTD, defined as sum of the diameter of target lesions) was $38.8 \pm 15.7 \mathrm{~mm}$ at baseline. $\mathrm{CT}$ results indicated that $40.0 \%$ of early tumor shrinkage was achieved 4 weeks after apatinib administration, with the TTD diminishing from $44.7 \pm 11.0$ to $29.6 \pm 8.0 \mathrm{~mm}(P<0.001)$ in 7 patients available for evaluation. Eight weeks later, the TTD shrank down to $22.8 \pm 8.1 \mathrm{~mm}(P=0.001$; the individual responses are shown in Figure 2B). Figure 3B shows a target lesion of the 76-year-old male patient, the diameter of which decreased from 21.3 $\mathrm{mm}$ to $15.9 \mathrm{~mm}$ and $9.5 \mathrm{~mm}$ after 4 and 8 weeks of treatment, respectively.
Eight weeks following apatinib administration, 9 patients achieved partial response (PR) and one patient achieved stable disease (SD), according to the RECIST 1.1 criteria (Table 1). The disease control rate (DCR) and objective response rate (ORR) of the study were $100 \%$ $(10 / 10)$ and $90 \%(9 / 10)$, respectively.

\section{Evaluation of glucose metabolic response of target lesions based on PET/CT}

Significant decrease in maximum standard uptake value (SUVmax) was revealed after 8 weeks of treatment, indicating a rapid decrease in glucose metabolism in the target lesions. The SUVmax diminished from $6.53 \pm 5.14$ to $2.56 \pm 1.67(P=0.032)$ and $2.45 \pm 1.48(P=0.020)$ after 4 and 8 weeks of treatment, respectively (the responses of each lesion are shown in Figure 2C). Especially, in the male patient mentioned above, the SUVmax decreased from 11.38 to 1.26 and 0.95 at the 4- and 8-week timepoint following apatinib treatment, respectively (Figure 3C).

\section{The correlation among $\mathrm{Tg}$, lesions diameter and SUVmax}

The decrease rate of Tg level, TTD and SUVmax 8 weeks after apatinib administration were compared within individual cases (Figure 2D). Tg exhibited the most and fastest decrease, followed by glucose metabolism revealed by PET/CT and TTD reflected by CT. The relationships among changes in the three indicators were 
further analyzed. No correlation was found in patients between the changes in Tg level and SUVmax (after 8 weeks) or Tg level and TTD (Figure 4A, 4B), while linear correlations of change in diameter after 8 weeks of therapy with changes in SUVmax after 4 and 8 weeks of therapy were observed in the target lesions $(P=0.009$ and 0.036 , respectively) (Figure 4C, 4D).

\section{Safety}

In this study, most patients suffered from grade 1 to grade 3 treatment-related adverse events (AEs), and no severe AE (SAE) related to apatinib was observed during the 8 weeks treatment period. The most common AE was hand-foot-skin reaction (HFSR) (9/10). No cardiovascular AE was reported. The grade 3 AEs
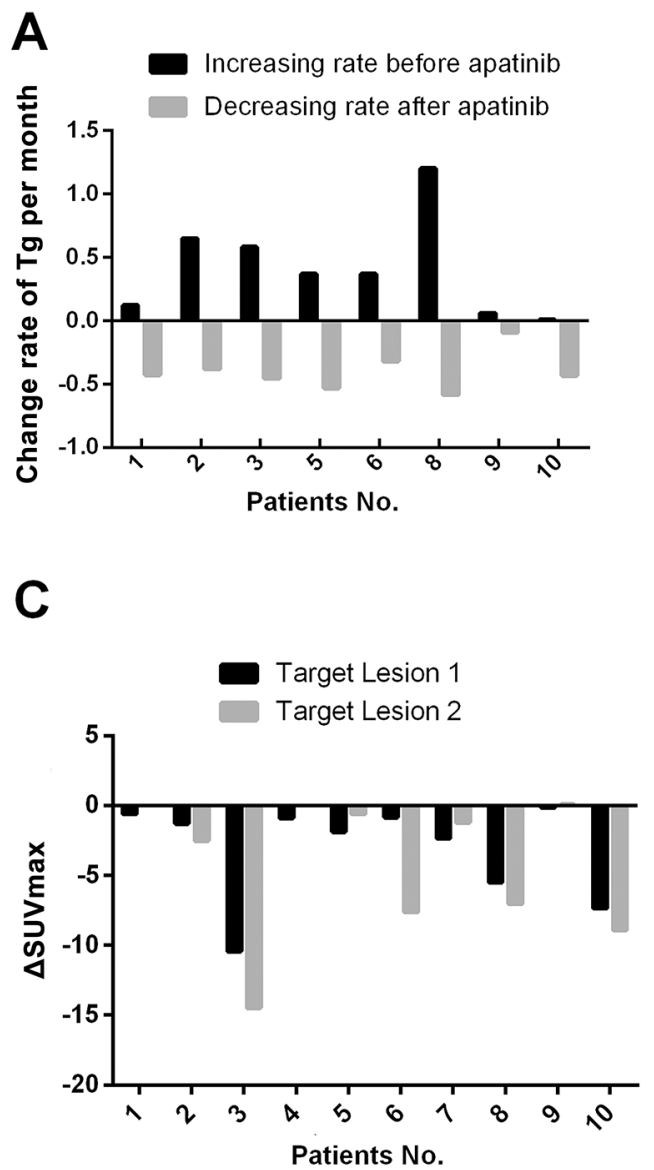

included HFSR, hypertension, hypocalcemia, proteinuria, neutropenia, thrombocytopenia, pharyngolaryngeal pain, and dysphagia, which accounted for $50 \%, 30 \%, 20 \%$, $10 \%, 10 \%, 10 \%, 10 \%$, and $10 \%$, respectively. Treatment suspension was required due to grade 3 HFSR, proteinuria, neutropenia, thrombocytopenia, or dysphagia. The details in AEs related to apatinib are shown in Table 2, with a comparison with those of sorafenib and lenvatinib as previously described $[1,7]$. AEs were listed if they were reported in more than $10 \%$ of patients.

\section{DISCUSSION}

Progress has been made in revealing the molecular mechanisms of thyroid cancer in the past 10 years [18], thus promoting clinical translational research in

B

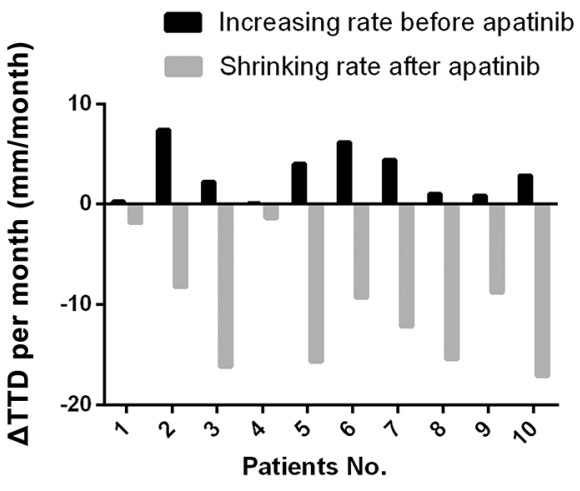

D

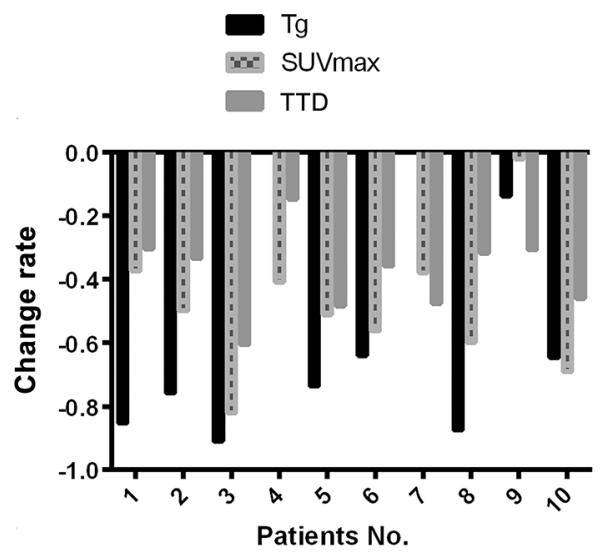

Figure 2: The changes in Tg, TTD, and SUVmax before and 8 weeks after apatinib therapy. A. The change rate of Tg per month in individual patients before and after apatinib therapy. The change rate of Tg per month was calculated according to the following formula: $\left(\mathrm{Tg}-\mathrm{Tg}_{\text {baseline }}\right) / \mathrm{Tg}_{\text {baseline }} /$ month. B. The change in sum of target lesion diameter per month in individual patients before and after apatinib therapy. The change in sum of target lesion diameter per month ( $\triangle \mathrm{TTD}$ per month) was calculated according to the following formula: (TTD-TTD ${ }_{\text {baseline }}$ )/month. C. The change in SUVmax of target lesions after apatinib therapy. The change in SUVmax was defined

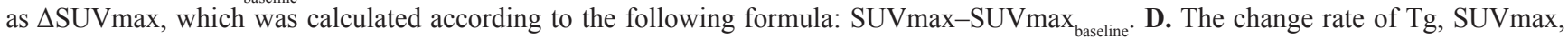
TTD in individual patient after 8 weeks of apatinib therapy. The change rate of $\mathrm{Tg}$ was calculated according to the following formula: $\left(\mathrm{Tg}-\mathrm{Tg}_{\text {baseline }}\right) / \mathrm{Tg}_{\text {baseline }}$. The change rate of SUVmax was calculated according to the following formula: (SUVmax-SUVmax $\left.{ }_{\text {baseline }}\right) /$ SUVmax $_{\text {baseline. }}$. The change rate of TTD was calculated according to the following formula: (TTD-TTD baseline $) / \mathrm{TTD}_{\text {baseline }}$. Tg, thyroglobulin; TTD, total tumor diameter which was defined as sum of the diameter of target lesions; SUVmax, maximum standard uptake value. 


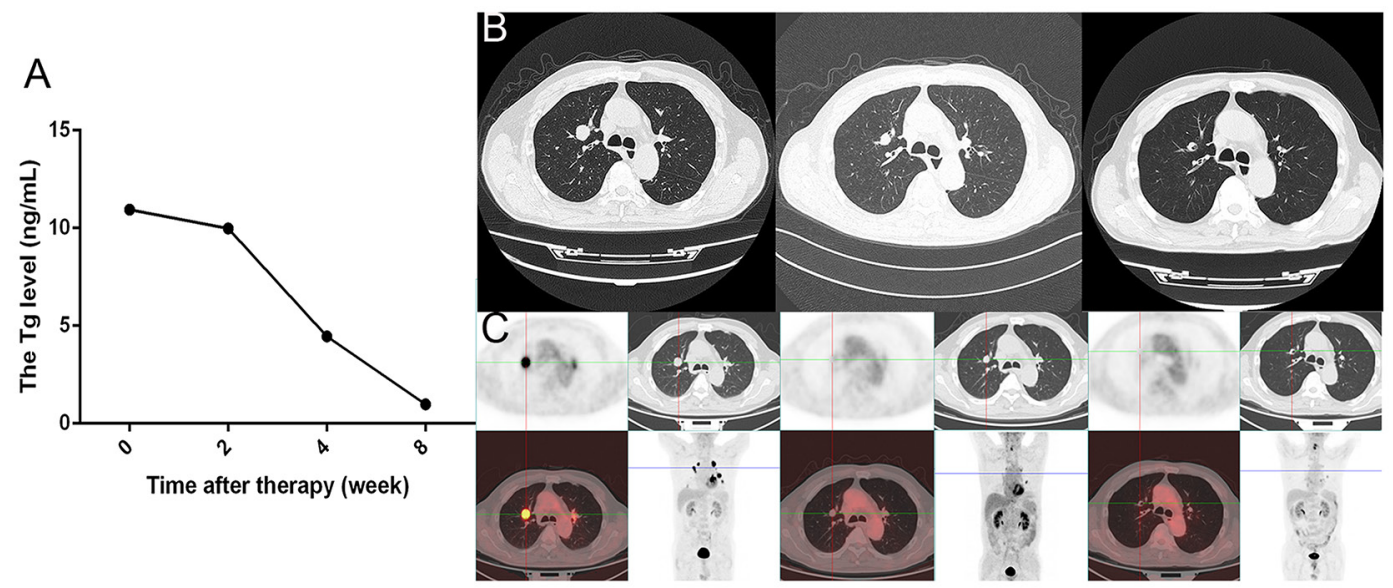

Figure 3: The Tg, CT, and PET/CT results obtained before and after apatinib therapy in a male patient. A. Tg levels at baseline and after therapy. B. CT images at baseline and after 4 and 8 weeks of apatinib therapy. C. PET/CT images at baseline and after 4 and 8 weeks of apatinib therapy. Tg, thyroglobulin; CT, computed tomography; PET/CT, positron emission tomography/ computed tomography.

A
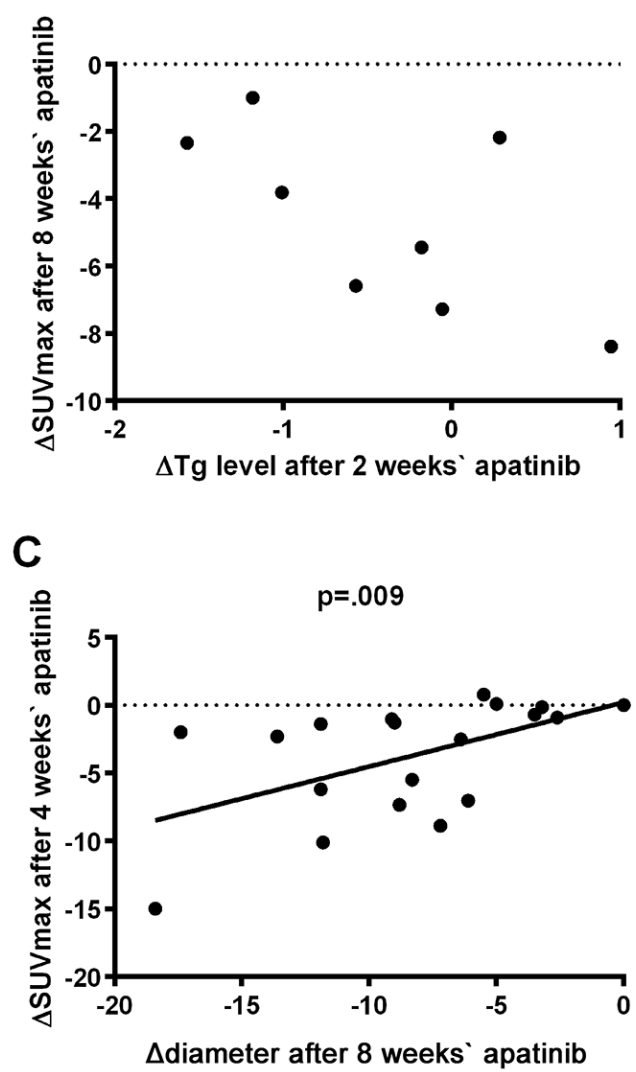

B

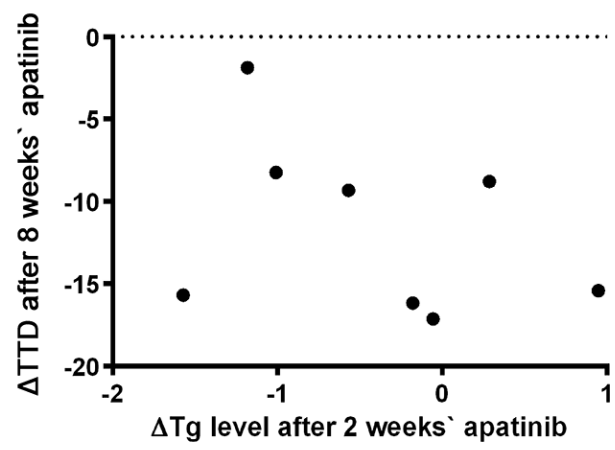

D

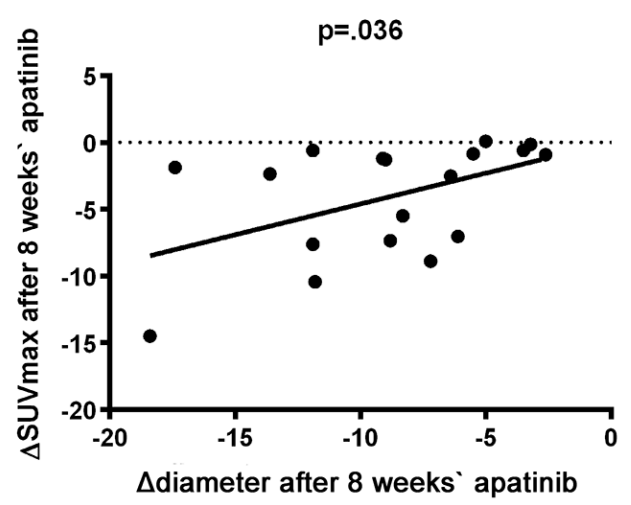

Figure 4: The association between Tg, TTD and SUVmax. A. Relationship between changes in SUVmax after 8 weeks of apatinib therapy and Tg after 2 weeks of apatinib therapy in patients. B. Relationship between changes in TTD after 8 weeks of apatinib therapy and Tg after 2 weeks of apatinib therapy in patients. C. Relationship between changes in SUVmax after 4 weeks of apatinib therapy and diameter after 8 weeks of apatinib therapy in target lesions. D. Relationship between changes in SUVmax after 8 weeks of apatinib therapy and diameter after 8 weeks of apatinib therapy in target lesions. $\Delta \mathrm{Tg}$ : $\mathrm{Tg}-\mathrm{Tg}_{\text {baseline }} ; \Delta \mathrm{TTD}$ : TTD-TTD diameter-diameter $_{\text {baseline }} ; \Delta \mathrm{SUVmax}$ : SUVmax-SUVmax baseline Tg, thyroglobulin; TTD, total tumor diameter which was defined as sum of the diameter of target lesions; SUVmax, maximum standard uptake value. 
Table 2: Adverse events

\begin{tabular}{lcccccc}
\hline Adverse events (\%) & \multicolumn{2}{c}{ Apatinib } & \multicolumn{2}{c}{ Lenvatinib [7] } & \multicolumn{2}{c}{ Sorafenib [1] } \\
\hline HFSR & All grades & Grade $\geq \mathbf{3}$ & All grades & Grade $\geq \mathbf{3}$ & All grades & Grade $\geq \mathbf{3}$ \\
Hypertension & 90 & 50 & 31.8 & 3.4 & 76.3 & 20.3 \\
Proteinuria & 80 & 30 & 67.8 & 41.8 & 40.6 & 9.7 \\
Elevated transaminase & 70 & 10 & 31.0 & 10 & NA & NA \\
Fatigue & 70 & 0 & NA & NA & $12.6 / 11.1$ & $2.9 / 1.0$ \\
Hypocalcemia & 70 & 0 & 59.0 & 9.2 & 49.8 & 5.8 \\
Hyperbilirubinemia & 70 & 20 & 6.9 & 2.7 & 18.8 & 5.8 \\
Dyspepsia & 40 & 0 & NA & NA & NA & NA \\
Mucositis oral & 40 & 0 & 10 & 0 & 31.9 & 2.4 \\
Dysgensia & 40 & 0 & 35.6 & 4.2 & 23.2 & 0.5 \\
Leukopenia & 40 & 0 & 16.9 & 0 & NA & NA \\
Neutropenia & 30 & 0 & NA & NA & NA & NA \\
Elevated GGT & 30 & 10 & NA & NA & NA & NA \\
Thrombocytopenia & 20 & 0 & NA & NA & NA & NA \\
Pharyngolaryngeal pain & 20 & 10 & NA & NA & NA & NA \\
Dysphagia & 20 & 10 & 10 & 0.4 & 10.1 & 0 \\
\hline
\end{tabular}

HFSR: hand-foot-skin reaction; GGT: gamma-glutamyl transpeptidase.

N/A: not available.

RAIR-DTC. The enhanced catalytic activity via the phosphorylation of many intracellular proteins involved in the signal transduction cascade is responsible for uncontrolled cell growth and thus is the molecular rationale for the use of TKIs in thyroid cancer. Several TKIs have been tested for the treatment of advanced, progressive DTC patient who no longer benefits from radioiodine therapy, with a PR rate varying from $8 \%$ to $64.7 \%$ [1, 7, 19-22]. Sorafenib is the first FDA-approved TKI, which targets VEGFR 1, 2, and 3, platelet-derived growth factor receptor (PDGFR), Raf-1, RET, and BRAF. It displayed a PR rate of $12.2 \%$. Lenvatinib, a TKI targeting VEGFRs, fibroblast growth factor receptors, and PDGFR, was the second TKI approved for its clinical use in RAIR-DTC. Lenvatinib achieved a reported PR rate of $64.7 \%$. In this pilot study, PR was achieved in $90 \%$ of patients after only 8 weeks of apatinib administration. Relatively, this is the best and fast PR rate, which had ever achieved in RAIR-DTC treated with TKIs. This rapid response also shed light on using apatinib as preoperative adjuvant therapy, which aims at quickly reducing the tumor burden and facilitating complete tumor resection. Though it remains unclear, the highly specific and selective inhibition of VEGFR-2 by apatinib potently represses VEGF-mediated endothelial cell migration and proliferation, which might be responsible for the majority of its high efficacy [23]. In vitro, enzyme experiments showed that apatinib (YN968D1) was a highly selective inhibitor of VEGFR-2 with an IC50 of $1 \mathrm{nM}$. The value is much lower than that of sunitinib $(5 \mathrm{nM})$, sorafenib (90 $\mathrm{nM})$ or even lenvatinib (4 $\mathrm{nM})$, indicating that apatinib can effectively inhibit the proliferation, migration and tube formation of human umbilical vein endothelial cell [23-25]. Besides, the difference between Chinese and Western population concerning genomic and demographic characteristics might also play a role [26-28].

The challenges to assess the response to TKI include finding suitable markers to reflect its early efficacy and timely identifying the patients who are unlikely to benefit from expensive TKI therapy that is not covered by Chinese health insurance and cannot be afforded by most of the Chinese families. Fortunately, Tg, an organ-specific glycoprotein that is produced only by normal thyroid or differentiated thyroid cancer cells, becomes a sensitive marker for the surveillance of thyroid cancer. Serum Tg was proven to be a specific marker for predicting distant metastases after surgery even without any imaging evidence before radioiodine ablation therapy in our previous work [29]. Also, it becomes a reliable marker for detecting DTC recurrence after successful remnant thyroid ablation $[30,31]$. All patients enrolled in this study achieved a successful thyroid remnant ablation after at least one 
time of radioiodine therapy. We noticed an immediate reduction of $\mathrm{Tg}$ level as early as 2 weeks after apatinib therapy, and a more pronounced $\mathrm{Tg}$ reduction can be observed compared with the simultaneous changes in TTD and glucose metabolism, implying that Tg could be used as both sensitive and convenient serum indicator in reflecting the early biochemical response to TKI in RAIRDTC. One patient presented a transient increase in $\mathrm{Tg}$ after 2 weeks of therapy but a dramatic decrease 4 weeks post-treatment, which may be caused by the probable $\mathrm{Tg}$ release due to the self-destruction of tumor cells in response to apatinib [32]. Considering this early change in Tg, we proposed that RECIST evaluation, which was indicated at least 2 to 3 months after TKI in most previous studies [19-22], might miss the probable early anatomic response in RAIR-DTC. CT evaluation at 4-week time-point in this study did identify more than $40.0 \%$ of initial mean shrinkage, which indicated an early partial response in 14 target lesions available for assessment. Thus for the first time, we demonstrated that RECIST assessment could be applied earlier than currently recommended for the purpose of early response identification. In addition, anatomic imaging alone using RECIST1.1 response criterion has the limitation in reflecting metabolism. Over recent years, there is an evolving consideration for ${ }^{18} \mathrm{FDG}-\mathrm{PET}$ assessment in solid tumors, which is gradually accepted as PERCIST 1.0 response criteria and proven to be a complementary assessment method in both clinical trials and structural quantitative clinical evaluation [33, 34]. Our study substantiated a dramatic decrease in SUVmax after 4 weeks of therapy using ${ }^{18}$ FDG-PET/CT, which was correlated to the decrease in target lesion diameter revealed by CT. Besides, more significant decrease in SUVmax was observed compared with the simultaneous structural change reflected by RECIST (60.8 \% vs 34.0\%), implying an earlier and more pronounced metabolic response prior to structural reaction in response to apatinib. Thus ${ }^{18} \mathrm{FDG}$ PET holds the promise to afford an on-going in-vivo biochemical evidence for the early assessment of response to TKI in RAIR-DTC. Would an earlier ${ }^{18}$ FDG PET be more informative for the treatment response assessment? Several studies demonstrated the promising application of ${ }^{18}$ FDG PET in the early evaluation of lung cancer treated with epidermal growth factor receptor (EGFR) TKI, with varied evaluation time, even as early as 2 days after drug administration [35, 36]. For thyroid cancer, researchers ever tried to perform the PET/CT after 7 days treated with sunitinib, but failed to capture its significant therapeutic effect [21]. This might be due to the different mechanism involved in TKI treatment. Early tumor necrosis suggested by the variation of $\mathrm{Tg}$ after 2 weeks of apatinib in this study might also be a confounding factor, which prevents an interpretation of an earlier PET evaluation less than 2 weeks. Thus, considering the influence factors on efficacy assessment, radiation exposure and safety, PET/CT at 4 weeks set in this study made sense and came out with both encouraging and convincing results. Significant falling of Tg achieved PR after 8 weeks of therapy, also indicating that monitoring Tg level could be a handy and useful method to detect the early response to TKI in RAIR-DTC. Our study showed no significant correlation between changes in $\mathrm{Tg}$ and SUVmax or TTD, yet, the possible reasons might be the limited numbers of cases, or the dedifferentiation of tumor partially losing the ability to produce Tg. In a word, the early evaluation modalities we adopted in this study revealed that the 4 weeks RECIST evaluation combined with Tg and glucose metabolism detection might be helpful in earlier identifying patients who unlikely response to those expensive and toxicity TKI and should withdraw from the unbeneficial therapy as early as possible.

In addition to the quick response and high efficacy of apatinib, acceptable and predictable toxicities were observed [16, 17]. HFSR was the most frequent AE during the therapy. As the most common AE of TKI, despite not life threatening, HFSR might seriously impair activities and daily life, even causing drug suspending and reduction [37]. In this study, $90.0 \%$ patients presented with HFSR, compared to $31.8 \%$ of lenvatinib and $76.3 \%$ of sorafenib in treating thyroid cancer $[1,7]$. Five of them $(50.0 \%)$ suffered from grade 3 HFSR (3.4\% of lenvatinib and $20.3 \%$ of sorafenib), resulting in therapy suspension at the third to the fifth week $[1,7]$. After drug discontinuation for one week, the HFSR receded to grade 1. All AEs were controllable and most of them were well tolerated. Though tolerable, patients with thyroid cancer seemed to experience more HFSR and grade 3 AEs compared with Li's phase III study in patients with advanced gastric cancer, who might present malabsorption by gastric surgery or by digestive tumor itself, implying that dose adjustment might be needed to balance the efficacy and adverse effect when treating RAIR-DTC [17].

To summarize, our results presented safe, rapid and high efficacy of apatinib in patients with RAIR-DTC. Along with RECIST evaluation, Tg and ${ }^{18} \mathrm{FDG}-\mathrm{PET} / \mathrm{CT}$ could afford more informative and distinct biochemical and glucose metabolism changes, and could be used as early response indicators. Further follow-up of this study are still underway, and the overall survival (OS), progression free survival (PFS), duration of response (DoR) will be reported.

\section{PATIENTS AND METHODS}

This study was approved by the institutional review board of Peking Medical College Hospital Ethics Committee, and was conducted in accordance with the Declaration of Helsinki and International Conference on Harmonization Good Clinical Practice guidelines. The informed consents were obtained from all patients before enrollment. This study is registered with ClinicalTrails. gov (number, NCT02731352). 


\section{Patients}

Patients aged 18 years or older with histologically confirmed locally advanced or metastatic RAIR-DTC were recruited in this study. The inclusion criteria included at least one progressive lesion according to the RECIST (version 1.1); disease progression within the past 14 months according to RECIST (version 1.1); and meet the following definition of RAIR-DTC. Patients were classified as RAIR-DTC if they met one of the following criteria: 1) had at least one measurable lesion without iodine uptake on ${ }^{131} \mathrm{I}$ scan, 2) had one measurable lesion that had progressed within the past 12 months even it could uptake radioiodine, or 3) received cumulative activity of ${ }^{131} \mathrm{I}$ over $600 \mathrm{mCi}$. All eligible patients had received no prior therapy with a TKI or chemotherapy.

\section{Study design and treatment}

Both efficacy and safety of apatinib (Jiangsu Hengrui Medicine, Lianyungang, China) were evaluated in this study. The study diagram is shown in Figure 1. All enrolled RAIR-DTC patients were commenced on apatinib at a dose of $750 \mathrm{mg}$ q.d. A treatment cycle was defined as 4 weeks. Treatment interruption caused by toxicities of apatinib was allowed for no more than 2 times or a maximum duration of 2 weeks (either continuously or cumulatively) in one cycle. Dose reduction to $500 \mathrm{mg}$ q.d. was accepted for only one time, and re-escalation was not accepted. Treatment continued until the disease progression, drug intolerance or withdrawal of consent from the study.

The primary endpoints were change in serum $\mathrm{Tg}$ concentration, DCR and ORR. The secondary endpoints included OS, PFS and DoR, changes in iodine metabolism, glucose metabolism and tumor angiogenesis, and safety. The present report described the early response of apatinib in RAIR-DTC, including change in Tg level, DCR, ORR, change in glucose metabolism in target lesions, and safety during 2 treatment cycles.

\section{Study assessments}

VEGF expression in the primary foci was detected by immunohistochemistry using Vectastain $A B C$ kit (Vector, Burlingame, USA). After pretreatment and blocking, deparaffinized section (3-mm) was incubated with primary antibody (Abcam Inc., Cambridge, USA) at $4^{\circ} \mathrm{C}$ overnight followed by secondary antibody at room temperature for $30 \mathrm{~min}$. The results were analyzed by two independent pathologists.

The serum $\mathrm{Tg}$ concentrations at baseline and every 2 weeks after treatment during the therapy were measured. Simultaneously, the $\mathrm{TgAb}$ was detected to identify potential assay interference. Measurements were performed by electrochemiluminescence immunoassay (Roche Diagnostics GmbH, Mannheim, Germany) with a functional sensitivity of $0.100 \mathrm{ng} / \mathrm{mL}$ and $10 \mathrm{IU} / \mathrm{mL}$, respectively. Change in $\mathrm{Tg}$ was defined as $\Delta \mathrm{Tg}$, which was calculated as $\mathrm{Tg}-\mathrm{Tg}_{\text {baseline }}$. The change rate of $\mathrm{Tg}$ was calculated as $\left(\mathrm{Tg}-\mathrm{Tg}_{\text {baseline }}\right) / \mathrm{Tg}_{\text {baseline }}$, and change rate per month was also calculated.

Tumor diameters were measured at baseline and every 4 weeks after treatment using CT. TTD was defined as the sum of the tumor diameter of each lesion. Change in TTD $(\triangle T T D)$ per month was calculated as (TTD-TTD ${ }_{\text {baseline }}$ )/ month, and change rate of TTD was calculated as (TTD$\mathrm{TTD}_{\text {baseline }} / \mathrm{TTD}_{\text {baseline }}$. Furthermore, tumor response was evaluated as defined by RECIST (version 1.1).

Additionally, ${ }^{18} \mathrm{~F}$-fluorodeoxyglucose $\left({ }^{18} \mathrm{FDG}\right)$ PET/CT scan was conducted at baseline and every 4 weeks after treatment to assess the glucose metabolic response to apatinib. Briefly, patients were instructed to avoid strenuous work or exercise for at least 24 hours and fast for more than 4 hours before the ${ }^{18} \mathrm{~F}-\mathrm{FDG}$ (0.15 mCi per kilogram) injection. After injection, patients were rested in a warm, darkened room for nearly 40 minutes. The acquisition was performed from skull to mid-thigh ( 5 to 6 beds position, $2 \mathrm{~min}$ per bed) using a Siemens Biograph 64 Truepoint TrueV PET/CT scanner. Images were reconstructed with manufacturer-provided software by using an ordered subset expectation maximization algorithm. SUVmax was used to evaluate the ${ }^{18} \mathrm{~F}$-FDG uptake in a lesion. Change in SUVmax was defined as $\triangle \mathrm{SUVmax}$, which was calculated as SUVmax-SUVmax baseline . The change rate of SUVmax was calculated as (SUVmaxSUVmax baseline $_{\text {e }}$ / SUVmax baseline, $_{\text {, }}$

Adverse events were assessed and recorded until 4 weeks after the last drug of the study was administered.

\section{Statistical analysis}

Categorical variables were expressed as number and percentage, and continuous variables were expressed as mean \pm standard deviation (SD) or median and interquartile range when appropriate. A two-sample t-test or Wilcoxon rank sum test was used to compare the tumor diameter before and after apatinib treatment. Correlation between two variables was analyzed by linear regression. SPSS (version 19.0; SPSS Inc, Chicago, IL, USA) and Prism 6 (GraphPad Software, San Diego, CA, USA) were used for statistical analyses. A $P$ value less than 0.05 was considered statistically significant.

\section{ACKNOWLEDGMENTS}

All authors thank the patients who participated in this study and HengRui Pharmaceuticals for providing apatinib free of charge.

\section{CONFLICTS OF INTEREST}

The authors have no conflicts of interest to declare. 


\section{FUNDING}

This work was supported by National Natural Science Foundation of China (Grant No 81571714).

\section{REFERENCES}

1. Brose MS, Nutting CM, Jarzab B, Elisei R, Siena S, Bastholt L, de la Fouchardiere C, Pacini F, Paschke R, Shong YK, Sherman SI, Smit JW, Chung J, et al. Sorafenib in radioactive iodine-refractory, locally advanced or metastatic differentiated thyroid cancer: a randomised, double-blind, phase 3 trial. Lancet. 2014; 384: 319-28. doi: 10.1016/S0140-6736(14)60421-9.

2. Chen W, Zheng R, Baade PD, Zhang S, Zeng H, Bray F, Jemal A, Yu XQ, He J. Cancer statistics in China, 2015. CA Cancer J Clin. 2016; 66: 115-32. doi: 10.3322/caac.21338.

3. Siegel RL, Miller KD, Jemal A. Cancer statistics, 2016. CA Cancer J Clin. 2016; 66: 7-30. doi: 10.3322/caac.21332.

4. Haugen BR, Alexander EK, Bible KC, Doherty GM, Mandel SJ, Nikiforov YE, Pacini F, Randolph GW, Sawka AM, Schlumberger M, Schuff KG, Sherman SI, Sosa JA, et al. 2015 American Thyroid Association Management Guidelines for adult patients with thyroid nodules and differentiated thyroid cancer: The American Thyroid Association Guidelines Task Force on thyroid nodules and differentiated thyroid cancer. Thyroid. 2016; 26: 1-133. doi: 10.1089/thy.2015.0020.

5. Schlumberger M, Brose M, Elisei R, Leboulleux S, Luster M, Pitoia F, Pacini F. Definition and management of radioactive iodine-refractory differentiated thyroid cancer. Lancet Diabetes Endocrinol. 2014; 2: 356-8. doi: 10.1016/ S2213-8587(13)70215-8.

6. Durante C, Haddy N, Baudin E, Leboulleux S, Hartl D, Travagli JP, Caillou B, Ricard M, Lumbroso JD, De Vathaire F, Schlumberger M. Long-term outcome of 444 patients with distant metastases from papillary and follicular thyroid carcinoma: benefits and limits of radioiodine therapy. J Clin Endocrinol Metab. 2006; 91: 2892-9. doi: $10.1210 /$ jc. $2005-2838$.

7. Schlumberger M, Tahara M, Wirth LJ, Robinson B, Brose MS, Elisei R, Habra MA, Newbold K, Shah MH, Hoff AO, Gianoukakis AG, Kiyota N, Taylor MH, et al. Lenvatinib versus placebo in radioiodine-refractory thyroid cancer. N Engl J Med. 2015; 372: 621-30. doi: 10.1056/ NEJMoa1406470.

8. Volante M, Rapa I, Gandhi M, Bussolati G, Giachino D, Papotti M, Nikiforov YE. RAS mutations are the predominant molecular alteration in poorly differentiated thyroid carcinomas and bear prognostic impact. J Clin Endocrinol Metab. 2009; 94: 4735-41. doi: 10.1210/ jc.2009-1233.

9. Ricarte-Filho JC, Ryder M, Chitale DA, Rivera M, Heguy A, Ladanyi M, Janakiraman M, Solit D, Knauf JA, Tuttle RM, Ghossein RA, Fagin JA. Mutational profile of advanced primary and metastatic radioactive iodinerefractory thyroid cancers reveals distinct pathogenetic roles for BRAF, PIK3CA, and AKT1. Cancer Res. 2009; 69: 4885-93. doi: 10.1158/0008-5472.CAN-09-0727.

10. Yang $\mathrm{K}$, Wang $\mathrm{H}$, Liang $\mathrm{Z}$, Liang J, Li F, Lin Y. BRAFV600E mutation associated with non-radioiodineavid status in distant metastatic papillary thyroid carcinoma. Clin Nucl Med. 2014; 39: 675-9. doi: 10.1097/ RLU.0000000000000498.

11. Klein M, Picard E, Vignaud JM, Marie B, Bresler L, Toussaint B, Weryha G, Duprez A, Leclere J. Vascular endothelial growth factor gene and protein: strong expression in thyroiditis and thyroid carcinoma. J Endocrinol. 1999; 161: 41-9.

12. Jing FJ, Liang J, Liang ZY, Meng C, Long W, Li XY, Lin YS. BRAF(V600E) mutation is not a positive predictor for distant metastasis in sporadic papillary thyroid carcinoma. Chin Med J (Engl). 2013; 126: 3013-8.

13. de Araujo-Filho VJ, Alves VA, de Castro IV, Lourenco SV, Cernea CR, Brandao LG, Ferraz AR. Vascular endothelial growth factor expression in invasive papillary thyroid carcinoma. Thyroid. 2009; 19: 1233-7. doi: 10.1089/ thy.2008.0179.

14. Zhao D, Jin X, Li F, Liang J, Lin Y. Integrin alphavbeta3 imaging of radioactive iodine-refractory thyroid cancer using 99mTc-3PRGD2. J Nucl Med. 2012; 53: 1872-7. doi: 10.2967/jnumed.112.107821.

15. Helal-Neto E, Brandao-Costa RM, Saldanha-Gama R, Ribeiro-Pereira C, Midlej V, Benchimol M, Morandi V, Barja-Fidalgo C. Priming endothelial cells with a melanoma-derived extracellular matrix triggers the activation of alphavbeta3/VEGFR2 axis. J Cell Physiol. 2016; 231: 2464-73. doi: 10.1002/jcp.25358.

16. Li J, Qin S, Xu J, Guo W, Xiong J, Bai Y, Sun G, Yang Y, Wang L, Xu N, Cheng Y, Wang Z, Zheng L, et al. Apatinib for chemotherapy-refractory advanced metastatic gastric cancer: results from a randomized, placebo-controlled, parallel-arm, phase II trial. J Clin Oncol. 2013; 31: 321925. doi: $10.1200 / J C O .2013 .48 .8585$.

17. Li J, Qin S, Xu J, Xiong J, Wu C, Bai Y, Liu W, Tong J, Liu Y, Xu R, Wang Z, Wang Q, Ouyang X, et al. Randomized, double-blind, placebo-controlled phase III trial of apatinib in patients with chemotherapy-refractory advanced or metastatic adenocarcinoma of the stomach or gastroesophageal junction. J Clin Oncol. 2016; 34: 1448-54. doi: 10.1200/JCO.2015.63.5995.

18. Xing M. Molecular pathogenesis and mechanisms of thyroid cancer. Nat Rev Cancer. 2013; 13: 184-99. doi: $10.1038 / \mathrm{nrc} 3431$.

19. Locati LD, Licitra L, Agate L, Ou SH, Boucher A, Jarzab B, Qin S, Kane MA, Wirth LJ, Chen C, Kim S, Ingrosso A, Pithavala YK, et al. Treatment of advanced thyroid cancer with axitinib: phase 2 study with pharmacokinetic/ pharmacodynamic and quality-of-life assessments. Cancer. 2014; 120: 2694-703. doi: 10.1002/cncr.28766. 
20. Bible KC, Suman VJ, Molina JR, Smallridge RC, Maples WJ, Menefee ME, Rubin J, Sideras K, Morris JC 3rd, McIver B, Burton JK, Webster KP, Bieber C, et al. Efficacy of pazopanib in progressive, radioiodine-refractory, metastatic differentiated thyroid cancers: results of a phase 2 consortium study. Lancet Oncol. 2010; 11: 962-72. doi: 10.1016/S1470-2045(10)70203-5.

21. Carr LL, Mankoff DA, Goulart BH, Eaton KD, Capell PT, Kell EM, Bauman JE, Martins RG. Phase II study of daily sunitinib in FDG-PET-positive, iodine-refractory differentiated thyroid cancer and metastatic medullary carcinoma of the thyroid with functional imaging correlation. Clin Cancer Res. 2010; 16: 5260-8. doi: 10.1158/1078-0432.CCR-10-0994.

22. Leboulleux S, Bastholt L, Krause T, de la Fouchardiere C, Tennvall J, Awada A, Gomez JM, Bonichon F, Leenhardt L, Soufflet C, Licour M, Schlumberger MJ. Vandetanib in locally advanced or metastatic differentiated thyroid cancer: a randomised, double-blind, phase 2 trial. Lancet Oncol. 2012; 13: 897-905. doi: 10.1016/S1470-2045(12)70335-2.

23. Tian S, Quan H, Xie C, Guo H, Lu F, Xu Y, Li J, Lou L. YN968D1 is a novel and selective inhibitor of vascular endothelial growth factor receptor-2 tyrosine kinase with potent activity in vitro and in vivo. Cancer Sci. 2011; 102: 1374-80. doi: 10.1111/j.1349-7006.2011.01939.x.

24. Wiegering A, Korb D, Thalheimer A, Kammerer U, Allmanritter J, Matthes N, Linnebacher M, Schlegel N, Klein I, Ergun S, Germer CT, Otto C. E7080 (lenvatinib), a multi-targeted tyrosine kinase inhibitor, demonstrates antitumor activities against colorectal cancer xenografts. Neoplasia. 2014; 16: 972-81. doi: 10.1016/j. neo.2014.09.008.

25. Wilhelm SM, Carter C, Tang L, Wilkie D, McNabola A, Rong H, Chen C, Zhang X, Vincent P, McHugh M, Cao Y, Shujath J, Gawlak S, et al. BAY 43-9006 exhibits broad spectrum oral antitumor activity and targets the RAF/MEK/ ERK pathway and receptor tyrosine kinases involved in tumor progression and angiogenesis. Cancer Res. 2004; 64: 7099-109. doi: 10.1158/0008-5472.CAN-04-1443.

26. Jin L, Chen E, Dong S, Cai Y, Zhang X, Zhou Y, Zeng R, Yang F, Pan C, Liu Y, Wu W, Xing M, Zhang X, et al. BRAF and TERT promoter mutations in the aggressiveness of papillary thyroid carcinoma: a study of 653 patients. Oncotarget. 2016; 7: 18346-55. doi: 10.18632/ oncotarget.7811.

27. Alzahrani AS, Alsaadi R, Murugan AK, Sadiq BB. TERT promoter mutations in thyroid cancer. Horm Cancer. 2016; 7: 165-77. doi: 10.1007/s12672-016-0256-3.

28. Kim TH, Park YJ, Lim JA, Ahn HY, Lee EK, Lee YJ, Kim KW, Hahn SK, Youn YK, Kim KH, Cho BY, Park DJ. The association of the BRAF(V600E) mutation with prognostic factors and poor clinical outcome in papillary thyroid cancer: a meta-analysis. Cancer. 2012; 118: 1764-73. doi: 10.1002/cncr.26500.
29. Lin $\mathrm{Y}, \mathrm{Li}$ T, Liang J, Li X, Qiu L, Wang S, Chen Y, Kang Z, Li F. Predictive value of preablation stimulated thyroglobulin and thyroglobulin/thyroidstimulating hormone ratio in differentiated thyroid cancer. Clin Nucl Med. 2011; 36: 1102-5. doi: 10.1097/ RLU.0b013e3182291c65.

30. Eustatia-Rutten CF, Smit JW, Romijn JA, van der KleijCorssmit EP, Pereira AM, Stokkel MP, Kievit J. Diagnostic value of serum thyroglobulin measurements in the follow-up of differentiated thyroid carcinoma, a structured meta-analysis. Clin Endocrinol (Oxf). 2004; 61: 61-74. doi: 10.1111/j.1365-2265.2004.02060.x.

31. Trevizam PG, Tagliarini JV, Castilho EC, de Alencar Marques M, Kiy Y, Mazeto GM. Thyroglobulin levels and thyroglobulin/thyrotropin ratio could predict the success of the ablative/therapeutic 131I in the differentiated thyroid cancers. Endocr Res. 2016: 1-7. doi: 10.3109/07435800.2016.1173056.

32. Stevic I, Dembinski TC, Pathak KA, Leslie WD. Transient early increase in thyroglobulin levels post-radioiodine ablation in patients with differentiated thyroid cancer. Clin Biochem. 2015; 48: 658-61. doi: 10.1016/j. clinbiochem.2015.04.009.

33. Yoon JW, Kim S, Kim SW, Kim YT, Kang WJ, Nam EJ. PET/CT response criteria (European Organization for Research and Treatment of Cancer) predict survival better than response evaluation criteria in solid tumors in locally advanced cervical cancer treated with chemoradiation. Clin Nucl Med. 2016; 41: 677-82. doi: 10.1097/ RLU.0000000000001269.

34. Wahl RL, Jacene H, Kasamon Y, Lodge MA. From RECIST to PERCIST: evolving considerations for PET response criteria in solid tumors. J Nucl Med. 2009; 50: 122S-50S. doi: 10.2967/jnumed.108.057307.

35. van Gool MH, Aukema TS, Hartemink KJ, Valdes Olmos RA, van Tinteren H, Klomp HM. FDG-PET/CT response evaluation during EGFR-TKI treatment in patients with NSCLC. World J Radiol. 2014; 6: 392-8. doi: 10.4329/wjr. v6.i7.392.

36. Takahashi R, Hirata H, Tachibana I, Shimosegawa E, Inoue A, Nagatomo I, Takeda Y, Kida H, Goya S, Kijima T, Yoshida M, Kumagai T, Kumanogoh A, et al. Early [18F] fluorodeoxyglucose positron emission tomography at two days of gefitinib treatment predicts clinical outcome in patients with adenocarcinoma of the lung. Clin Cancer Res. 2012; 18: 220-8. doi: 10.1158/1078-0432.CCR-11-0868.

37. Ren Z, Zhu K, Kang H, Lu M, Qu Z, Lu L, Song T, Zhou W, Wang H, Yang W, Wang X, Yang Y, Shi L, et al. Randomized controlled trial of the prophylactic effect of urea-based cream on sorafenib-associated hand-foot skin reactions in patients with advanced hepatocellular carcinoma. J Clin Oncol. 2015; 33: 894-900. doi: 10.1200/ JCO.2013.52.9651. 